\title{
Another Solution to the Problem of Theoretical Terms
}

\author{
Holger Andreas \\ Institute for Philosophy, Logic, and Theory of Science - LMU Munich
}

May 3, 2009

\begin{abstract}
In this paper, a solution to the problem of theoretical terms is developed that is based on Carnap's doctrine of indirect interpretation of theoretical terms. This doctrine will be given a semantic, model-theoretic explanation that is not given by Carnap himself as he remains content with a syntactic explanation. From that semantic explanation, rules for the truth-value assignment to postulates, i. e. sentences that determine the meaning of theoretical terms, are derived. The logical status of postulates will be clarified thereby in such a way that the problem of theoretical terms disappears.
\end{abstract}

Keywords: Model-theory, statement view, structuralist theory of science, theoretical terms.

\section{Introduction}

In structuralism, the problem of theoretical terms serves as a major objection to the statement-view in philosophy of science, i. e. the view that scientific theories may well be reconstructed as sets of statements. In essence, the problem is a semantical circle that concerns the relation between the meaning of theoretical terms and the meaning of axioms in which these terms occur. This circle appears to have no solution if the axioms of a scientific theory are seen as sentences or statements in the sense of predicate logic. By contrast, the problem of theoretical can be circumvented if the Ramsey sentence of a theory is chosen to represent that theory. This strategy has been pursued and further developed by Sneed in his The Logical Structure of Mathematical Physics (1979).

It is, I think, beyond doubt that with the structuralist framework a great advancement has been achieved in comparison to earlier attempts at logical reconstruction of scientific theories, as given by Carnap, Nagel, Braithwaite and others. In the present paper, I will nevertheless show that the structuralist critique of the statement view is based on assumptions which are not in accordance 
with Carnap's doctrines concerning the semantics of theoretical terms. His doctrine of indirect interpretation of theoretical terms requires rather a modification of the truth-conditional semantics to which Carnap himself, incoherently, adheres in his work on predicate logic since the Introduction to Semantics (1942). Such a modification will be developed in the present paper. The problem of theoretical terms will thereby be given another solution, in addition to what is called the Ramsey solution. Finally, the equivalence of the two solutions will be shown. At another place I hope to show that the solution developed here is also of interest from the perspective of structuralism.

\section{Exposition of the Problem}

Considered are scientific theories which are given in a more or less explicit axiomatic formulation. Sneed observes that there is a plethora of theories for which the following is true:

i) There is at least one term $t$ such that the values of $t$ can be determined iff the truth of sentences which are axioms of a theory $T$ is assumed. Let $\Phi$ be designating the set of these sentences; $t$ is either a predicate or a function symbol but not a constant.

ii) The truth-values of the sentences of $\Phi$ are determinable iff the values of the term $t$ are already given.

In light of this observation the following criterion is set up:

D 1. A Term $t$ is theoretical with respect to a theory $T$, or T-theoretical, iff there is no application of $T$ in which the value of $t$ can be determined independently from the axioms of $T$. An application of $T$ is an application of certain axioms of $T$ to a system of empirical entities.

In short, the problem of theoretical terms consists in a mutual dependency between the extension of a term and the truth-values of several axioms of a theory. Why is this threatening the cogency of a scientific theory? Now, the application of the axiomatic apparatus of a theory $T$ to a system of empirical objects is to be understood as implying the claim that certain axioms of $T$ are true of a non-empty set of empirical objects. Suppose that the axioms of a theory $T$ contain one or several terms which are T-theoretical. It follows then that there is a term $t$ whose values can only be determined with the help of sentences of $\Phi$. Assume furthermore that $t$ is specific to $T$ in such a manner that every application of $T$ implies an assertion regarding the values of $t$. (This assumption has been shown to apply to several theories.) The problem of theoretical terms then arises from the question of how an application of $T$ can ever be justified. This is because justifying the application of a theory requires one to know whether the axioms of $T$ are true in the respective application. But the truthvalues of these axioms are dependent on the values of $t$. And these values can, due to the theoretical character of $t$, only be determined if the axioms of $T$ are 
assumed to be true. We are thus captured in an epistemological circle when we attempt to justify the application of certain axioms of $T$ to a system of empirical entities.

The circle described is not only an epistemological one but also a semantic one, provided we view our methods of determining the extension of a linguistic expression as constituting its meaning. Then, the mutual dependency between the extension of $t$ and the extension of the sentences of $\Phi$ makes it difficult, if not impossible, to give a clear account of the meaning of $t$ and of the meaning of the sentences of $\Phi$.

The problem of theoretical terms may be exemplified at the measurement of the force function with the help of Hooke's law. This law goes as follows:

$$
\text { (2) } \mathbf{F}=-k\left(\mathbf{x}_{1}-\mathbf{x}_{0}\right) \text {, }
$$

where $\mathbf{F}$ stands for the force vector acting upon a coil spring, $\mathbf{x}_{1}$ and $\mathbf{x}_{0}$ are spatial vectors of the movable side of the coil spring where a force may impact, causing an elongation. $\mathbf{x}_{0}$ is the vector when there is no force acting, and $\mathbf{x}_{1}$ the vector when the force $\mathbf{F}$ is acting. The constant $k$ is dependent on particular properties of the coil spring. Assume that the value of a certain force is measured by a spring balance. Then, since such a measurement rests on the validity of Hooke's law, we have to assume that this law is valid in the measurement procedure. However, if we attempt to justify this application of Hooke's law, the value of the force function which ought to be measured would have to be known to us in advance. This situation appears to be inadmissible.

It would be no solution to the problem under consideration if the value of the force function is determined by another law of classical mechanics, say by the law of gravitation or Newton's second law. Such a strategy would only postpone the problem, but would not solve it. Nor would it be appropriate to interpret Hooke's law as a definition of the force function. Such a presumed definition would cover only a limited range of applications of classical mechanics, and could therefore not serve as a general definition of force. Finally, it is important to note that, in the existing expositions of classical mechanics, no technique of measurement for the force function can be found which does not depend on some law of this theory. ${ }^{1}$

One may object to the present exposition of the problem of theoretical terms that it is given in an empiricist and antirealist framework, thereby suggesting to dissolve that problem by simply abandoning the framework in which it is formulated. To this I reply that although the Ramsey solution qualifies as an empiricist position, it is far from clear whether Sneed's exposition of the problem rests on empiricist assumptions already. As shown here, this exposition is mainly based on the observation that there are linguistic expressions in the axioms of scientific theories whose extension can only be determined with the help of such axioms. This observation does not imply a commitment to empiricism or

\footnotetext{
${ }^{1}$ For the original exposition of the problem see Sneed (1979), pp. 31-40.
} 
antirealism in an obvious way. ${ }^{2}$

\section{The Ramsey Solution}

The strategy Sneed pursued to solve the problem of theoretical terms is based on the Ramsey sentence of a theory. The explanation of the meaning and structure of the Ramsey sentence requires some preliminaries concerning the dual-level conception of scientific language. Essential to this conception is the subdivision of the language into an observational and a theoretical level. For this subdivision I will assume the following explanations:

E 1. An expression belongs to the observational level if the determination of its extension, or at least a part of its extension, can proceed in a direct manner. In this case there is a method of determination which does not depend on general axioms or rules of inference which are assumed to be valid.

E 2. An expression belongs to the theoretical level if every method of determining its extension, or at least a part of its extension, rests on inferences. The validity of these inferences is dependent on general axioms or rules of inference which are neither true for logical reasons nor true in virtue of being a definition.

Although these explanations are not explicitly stated by Carnap, they appear to elucidate the semantic distinction between the two levels of the language in

\footnotetext{
${ }^{2}$ The problem of theoretical terms has received surprisingly little attention in the context of the debate on realism and antirealism. I may contemplate shortly on the question of whether it has some bearing on this debate. Antirealism may be defined as the view that the meaning of a linguistic expression is identified with our means of determining the extension of that expression. (This definition goes back to Dummett (1978), p. 146. That the focus of Dummett's explanation is on sentence meaning but not on the meaning of linguistic expressions in general seems inessential to our discussion.) Let us assume that scientific terms such as "force" in classical mechanics do have sense, where the sense, or intensional meaning, of a linguistic expression is what determines its extension. In the context of the present discussion the question arises of whether we shall account for the sense of a theoretical term in an antirealist or realist fashion. The antirealist will clearly refer to the axioms of $T$ as these are essential to our means of determining the extension of theoretical terms. By contrast, it is far from clear how a realist account of the sense of theoretical terms may look like. I am not aware of a satisfying answer to this question to be found in the literature. In particular, possible worlds semantics seem of limited help as these semantics take the extension of scientific terms as something that is already given. Rejecting the assumption that scientific terms do have sense seems not promising either because a purely extensional account of the meaning of scientific terms has its own shortcomings. For example, such an account would require a student of physics first to know the extension of the expression "force" in order to understand the meaning of that expression. By contrast, the teaching of physics and other natural sciences focuses rather on axioms and general explanations of how the extension of scientific terms can be determined when such terms are introduced.

Of course, these remarks are insufficient by far to rule out a realist approach to the meaning of theoretical terms. But they seem to indicate that the problem of theoretical terms is potentially apt to be used in a strong case for antirealism. For this reason we should not be surprised if the solutions proposed to this problem remain in an antirealist framework. Needless to say, there is a large number of philosophers of science who disagree totally with an antirealist approach to scientific theories. I am, however, not going to discuss metaphysical issues in the remainder of the paper.
} 
an appropriate manner. Note that the criterion following from the explanations is applicable not only to formal systems but also to the non-formalized usage of language. In a more extended discussion it could be shown that several arguments against the cogency of the distinction between observational and theoretical language, prominently raised by Kuhn and Feyerabend, are not sufficient to refute the assumption of a semantically stable observational language having an interpretation which does not depend on any axiomatic theory of the natural sciences. (See Andreas (2007).)

The distinction between two levels of the language gives rise to the following, well known distinction among the axioms of a theory:

i) C-postulates containing observational as well as theoretical terms.

ii) T-postulates containing only theoretical terms as non-logical symbols.

The set of T-postulates is also called the pure theory, whereas the set-theoretic sum of C- and T-postulates is called the interpreted theory. Among the Tpostulates there are general sentences which are considered to be laws of nature in a non-technical sense.

Some notational conventions need to be introduced at this point. $V_{o}$ is the set of non-logical symbols of the observational language, whereas $V_{t}$ is the set of non-logical symbols of the theoretical language. $\operatorname{Sent}\left(L\left(V_{o}\right)\right)$ designates the set of well formed sentences of the observational language, $\operatorname{Sent}\left(L\left(V_{o}, V_{t}\right)\right)$ those of the entire language containing $V_{o}$ and $V_{t}$ symbols. Let this system contain a sign for the identity between individual terms, i. e. terms designating individuals. $\operatorname{Sent}\left(L\left(V_{o}, V_{t}\right)\right) \backslash \operatorname{Sent}\left(L\left(V_{o}\right)\right)$ stands for the set of sentences containing at least one theoretical term. $\operatorname{TC}\left(t_{1}, \ldots, t_{n}, n_{1}, \ldots, n_{k}\right)$ stands for the conjunction of T- and C- postulates, where $t_{1}, \ldots, t_{n}$ are theoretical predicates or theoretical function symbols, and $n_{1}, \ldots, n_{k}$ predicates or function symbols of the observational language. It is important to note that, at least in Carnap's and Ramsey's version of the dual-level conception, there are no non-logical terms at the theoretical level designating individuals. Carnap assumes that there are individual constants at the theoretical level which are designating the natural numbers, yet he considers these symbols as logical ones. (See Carnap (1934), p. 255 or Carnap (1937), p. 327.)

The Ramsey sentence of a theory $T C$ results from the following transformations. In the first step, the predicates and function symbols $t_{1}, \ldots, t_{n}$ are replaced in $\mathrm{TC}$, the conjunction of postulates, by appropriate second order variables. In the second step, these variables are bound by existential quantifiers. As result we receive $T C^{R}$, the Ramsey sentence of a theory TC:

$$
\text { (2) } \exists X_{1} \ldots \exists X_{n} T C\left(X_{1}, \ldots, X_{n}, n_{1}, \ldots, n_{k}\right) \text {. }
$$

In Words: There are extensions of the theoretical terms for which the postulates $T C$ are true sentences. 
To what extent is it justified to view the Ramsey sentence as a solution to the problem of theoretical terms? There are two reasons for claiming this. The first reason is that the Ramsey sentence $T C^{R}$ and the original theory $T C$ are deductively equivalent with respect to their observational consequences, i. e. every sentence which can be derived from $T C$ is also derivable from $T C^{R}$. The following proposition is therefore valid: ${ }^{3}$

(3) For every $L\left(V_{o}\right)$ sentence $\phi: T C \vdash \phi$ iff $T C^{R} \vdash \phi$.

With respect to the predictions and retrodictions at the observational level there is thus no difference between the original theory and its Ramsey-sentence. From an empiricist point of view the Ramsey sentence appears therefore to be a fully satisfying surrogate for the original theory.

The second, even more important reason in favour of the Ramsey solution is that, in the Ramsey sentence, the axioms of the theory do not have the status of true propositions in the sense of predicate logic any more. By the Ramseysentence it is rather claimed that there are extensions of the theoretical terms satisfying the axioms of $T C$. Such a claim implies no assertion regarding the truth of these axioms. It is thus not required to justify the truth of any single axiom in order to justify the truth of the Ramsey sentence, which implies that the problem of theoretical terms does not occur.

Expounding the problem of theoretical terms and showing how it can be solved by the Ramsey account is not the sole objective of Sneed's (1971). Rather, he points out what he regards as several defects of the Ramsey view and henceforward develops the emended Ramsey view, which is the starting point of the structuralist account. The solution to the problem of theoretical terms to be developed here will be shown to be equivalent to the Ramsey view but not to the emended Ramsey view. For an attempt to develop an account based on the dual-level conception which is equivalent to the emended Ramsey view see Andreas (2007).

\section{Indirect Interpretation of Theoretical Terms}

In preparation of a second solution to the problem of theoretical terms I may argue for the following proposition:

$\mathbf{P}$ 1. The problem of theoretical terms arises iff a direct interpretation of these terms is assumed. By contrast, in Carnap's version of the dual-level conception, the theoretical terms are provided with an indirect interpretation.

The distinction between direct and indirect interpretation stems from Carnap's logic of science and stands in the need of further explanation:

E 3. The interpretation of a symbol is direct iff it is given by expressions of the meta-language and consists in an assignment of an intension or extension.

\footnotetext{
${ }^{3}$ For proof see Tuomela (1973), pp. 57-58.
} 
E 4. The interpretation of a symbol is indirect iff it is given by sentences of the object language which have the status of valid non-logical axioms in the calculus.

The distinction may be exemplified at a simple example. The predicates " $R$ " and "A" are interpreted in a direct manner by so-called rules of designation:

(4) The predicate " $R$ " designates the property of being rational.

(5) The predicate "A" designates the property of being an animal.

By contrast, the predicate " $\mathrm{H}$ " is interpreted indirectly by the following definition:

(6) $\quad \forall x(H(x) \leftrightarrow R(x) \wedge A(x))$.

Sentences of the object language which interpret symbols of this language have a particular status. Their truth-value is - in contrast to every other logical complex sentence - neither dependent on the extension of the non-logical symbols nor dependent on the valuations of the variables of the language. Rather their function consists in determining the extension of those non-logical symbols which are introduced into the language in an indirect manner. In Carnap's logic of science there are two kinds of indirect interpretation, one by definition and another by postulates. This distinguishes his account from most other predicate logic-based-accounts of scientific language. ${ }^{4}$

How can we understand the indirect interpretation of a symbol by postulates? Carnap confines himself to giving an explanation of the syntactic function of postulates: Postulates are, according to Carnap, non-logical axioms which are taken to be valid in the calculus and which therefore can be used in every derivation. An intuitive understanding of postulates is not required in order to justify their use in the calculus. The theoretical part of the calculus is rather 'free floating' and connected with the empirical world only by the interpretation of the observational terms. (See again Carnap (1939), pp. 67-69.)

I will now, in addition to Carnap's syntactic explanation, move the focus onto the semantic function of postulates. Some insight regarding this may be derived from an explanation of the semantic function of a definition. Based on the exposition of Beth's definability theorem, as given in standard accounts of mathematical logic, we can explain the interpretation of a term by a definition as follows:

E 5. Semantically seen, a set $\Phi_{d}$ of sentences that defines a non-logical symbol $P$ in a language $L(V)$ does impose a constraint on the admissible interpretations of $L(V \cup\{P\})$. This means, in terms of model-theoretic semantics, every admissible structure of the language $L(V \cup\{P\})$ must satisfy $\Phi_{d}$. With respect to a given

\footnotetext{
${ }^{4}$ For an explicit statement concerning the doctrine of indirect interpretation of theoretical terms see Carnap (1939), pp. 65-69 and Carnap (1956), p. 46n.
} 
$L(V)$ structure $\mathfrak{A}$ there is only one $L(V \cup\{P\})$ structure that expands $\mathfrak{A}$ such that $\Phi_{d}$ is satisfied. Thus, there is a unique interpretation of $P$ due to the conjoined constraint by $\Phi_{d}$ and $\mathfrak{A}$.

In what respects needs this explanation to be modified to account for the semantic function of postulates? From the expositions given in Carnap (1939), (1956), and (1958) it is apparent that the interpretation of theoretical terms by postulates is a rather holistic affair in the sense that a set of postulates interprets a set of theoretical terms. As a consequence of this, there is no oneto-one correspondence between a symbol and a set of sentences that interprets that symbol. It should furthermore not be assumed that the interpretation of theoretical terms results in a unique determination of the extension of these terms. $^{5}$

Yet another difference between definitions and postulates is that the introduction of theoretical terms by postulates may be accompanied by the introduction of another, theoretical domain of interpretation, in addition to the domain of interpretation for the observational language $L\left(V_{o}\right)$. In the case of Carnap's dual-level conception a domain of (mathematical) theoretical entities is assumed for the interpretation of the $V_{t}$ symbols, while in the case of Ramsey's conception $V_{o}$ and $V_{t}$ symbols have the same domain of interpretation. Taking these differences into account when observing the semantic similarities between definitions and postulates may result in the following explanation of the semantic function of postulates:

E 6. Semantically seen, a set $\Phi_{T C}$ of postulates that interprets a set of theoretical terms $V_{t}$ on the basis of a language $L\left(V_{o}\right)$ does impose a constraint on the admissible interpretations of the language $L\left(V_{o}, V_{t}\right)$. This means, in terms of model-theoretic semantics, every admissible $L\left(V_{o}, V_{t}\right)$ structure must satisfy $\Phi_{T C}$. The admissible $L\left(V_{o}, V_{t}\right)$ structures may have two domains of interpretation, one observational domain $D_{o}$ and a domain of theoretical entities $D_{t}$. With respect to a given $L\left(V_{o}\right)$ structure $\mathfrak{A}$ there may be several $L\left(V_{o}, V_{t}\right)$ structures that extend $\mathfrak{A}$ and satisfy $\Phi_{T C}$.

Since the interpretation of the symbols $V_{o}$ is assumed to be fixed by a given direct interpretation, there is a twofold imposition on the interpretation of theoretical terms, one by the interpretation of $L\left(V_{o}\right)$ and another by the postulates $T C$. This twofold imposition on the values of theoretical terms may be visualized by the following figure:

\footnotetext{
${ }^{5}$ Carnaps dictum that the interpretation of theoretical terms necessarily remains open to further strengthening seems to imply that the interpretation of a theoretical term by postulates does not amount to a unique determination of the extension of that term. For a closer examination of this point see Andreas (2007), p. 157.
} 


\section{Non-logical symbols of $V_{t}$}

Theoretical language
Indirect interpretation by postulates

Constraint on the admissible valuations of the $V_{t}$ symbols and the $L\left(V_{o}, V_{t}\right)$ sentences

\section{postulates}

Observational language
Non-logical symbols of $V_{O}$

Observational language

If a sentence $\phi$ is a postulate of the theory, then the truth-value assignment to $\phi$ belongs to the interpretation of the theoretical terms and is therefore not the result of a preceding direct interpretation. Hence, the truth-value assignment to postulates requires no information concerning the extensions of theoretical terms. This is the decisive argument in favour of proposition P1, the central claim of this section. ${ }^{6}$

\section{The Truth-Value Assignment to Theoretical Sentences}

The above figure is apt to provide an intuitive understanding of the notion of an indirect interpretation. However, precise rules for the truth-value assignment to theoretical sentences are, of course, not yet derivable from it. Such rules are being developed now. I will start from a general explanation of what it means that a set of sentences which have determinate truth values imposes a constraint on the admissible valuations of other sentences:

E 7. $\Phi_{1}$ and $\Phi_{2}$ are disjoint sets of sentences. $\nu_{1}: \Phi_{1} \rightarrow\{T, F\}$, i.e. $\nu_{1}$ is a function mapping sentences onto truth-values. $\Phi_{1, c}=\left\{\phi \mid \nu_{1}(\phi)=T\right\} \cup$ $\left\{\neg \phi \mid \nu_{1}(\phi)=F\right\} . \Phi_{1, c}$ is the conjunction of the set of sentences containing every true sentence of $\Phi_{1}$ and the set containing the negation of every false sentence of $\Phi_{1}$. A constraint on the admissible valuations of sentences of $\Phi_{2}$ is imposed on by the values of sentences belonging to $\Phi_{1}$. Then we say that any valuation $\nu_{2}(\phi), \nu_{2}: \Phi_{2} \rightarrow\{T, F\}$ satisfying the following rules is called an admissible valuation:

\footnotetext{
${ }^{6}$ It has to be admitted that there are versions of the dual-level conception in which a direct interpretation of theoretical terms is assumed; this is the case with the ones of Hempel (1958) and Tuomela (1973). Carnap's version is nevertheless, according to his own explanations, bound to an indirect interpretation of theoretical terms. (See Carnap (1939) pp. 65-69 and Carnap (1956), p. 46n.)
} 
i) $\nu_{2}(\phi)=\mathrm{T}$ iff the affirmation of $\phi$ is compatible with $\Phi_{1, c}$ in the sense that $\Phi_{1, c} \cup\{\phi\}$ is satisfiable.

ii) $\nu_{2}(\phi)=\mathrm{F}$ iff the negation of $\phi$ is compatible with $\Phi_{1, c}$ in the sense that $\Phi_{1, c}$ $\cup\{\neg \phi\}$ is satisfiable.

These conventions give only a unique determination of the value of a sentence $\phi$ if the affirmation of $\phi$ is admissible and the negation of $\phi$ is not or vice versa. To put it formally:

E 8. $\Phi_{1}, \Phi_{1, c}, \Phi_{2}, \nu_{1}$ and $\nu_{2}$ are like in E7. Then the sentences of $\Phi_{2}$ are to be valuated according to the following rules:

i) $\nu_{2}(\phi)=\mathrm{T}$ due to the imposition by $\Phi_{1}$ iff $\Phi_{1, c} \cup\{\phi\}$ is satisfiable and $\Phi_{1, c} \cup$ $\{\neg \phi\}$ is not.

ii) $\nu_{2}(\phi)=\mathrm{F}$ due to the imposition by $\Phi_{1}$ iff $\Phi_{1, c} \cup\{\neg \phi\}$ is satisfiable and $\Phi_{1, c}$ $\cup\{\phi\}$ is not.

These conventions may now be applied to the interpretation of theoretical sentences. According to the above explanation, the admissible values of sentences containing theoretical terms are limited firstly by the postulates TC and secondly by the values of the observational sentences. The values of the latter can be represented by the set $\Phi_{o}$ containing every true sentence of $L\left(V_{o}\right)$. $\Phi_{T C}$ is the set of postulates. Applying E8 to the valuation of theoretical sentences we receive:

E 9. $\nu_{t}(\phi)$ is a partial valuation for sentences of $\operatorname{Sent}\left(L\left(V_{o}, V_{t}\right)\right) \backslash \operatorname{Sent}\left(L\left(V_{o}\right)\right) \backslash$ $\Phi_{T C}$.

i) $\nu_{t}(\phi)=\mathrm{T}$ iff $\Phi_{T C} \cup \Phi_{o}=\phi$ and $\Phi_{T C} \cup \Phi_{o} \cup\{\phi\}$ is satisfiable.

ii) $\nu_{t}(\phi)=\mathrm{F}$ iff $\Phi_{T C} \cup \Phi_{o} \models \neg \phi$ and $\Phi_{T C} \cup \Phi_{o} \cup\{\neg \phi\}$ is satisfiable.

These rules leave open what the value of a sentence $\phi$ is if one of the following two cases occurs:

i) Both $\Phi_{T C} \cup \Phi_{o} \cup\{\phi\}$ and $\Phi_{T C} \cup \Phi_{o} \cup\{\neg \phi\}$ are satisfiable.

ii) Neither $\Phi_{T C} \cup \Phi_{o} \cup\{\phi\}$ nor $\Phi_{T C} \cup \Phi_{o} \cup\{\neg \phi\}$ is satisfiable.

Hence, we are to be prepared that there are truth-value gaps at the theoretical level which require a certain deviation from classical logic. The most convenient way to deal with such truth-value gaps appears to be the supervaluation logic developed by Bas van Fraassen and Graham Priest. Priest (2001), pp. 131-134, defines the set of supervaluations with respect to a partial classical valuation in the following manner:

D 2. $\operatorname{Sent}\left(L_{1}\right)$ stands for the set of sentences of a language $L_{1}$. $\nu_{p}: \Phi_{p} \rightarrow$ $\{T, F\}$, where $\Phi_{p}$ is a subset of $\operatorname{Sent}\left(L_{1}\right)$. Every valuation $\nu_{s}$ satisfying the following conditions is a supervaluation with respect to $\nu_{p}$ :

i) $\nu_{s}: \operatorname{Sent}\left(L_{1}\right) \rightarrow\{T, F\}$.

ii) $\nu_{s}(\phi)=\nu_{p}(\phi)$ for every $\phi \in \Phi_{p}$.

iii) $\left\{\phi \mid \nu_{s}(\phi)=\mathrm{T}\right\}$ is satisfiable. 
According to the second condition, every supervaluation $\nu_{s}$ must agree with $\nu_{p}$ in the domain of sentences where $\nu_{p}$ is defined. Those sentences whose values are not determined by $\nu_{p}$ are assigned to arbitrary values. Condition iii) ensures that the truth-rules for the sentential connectives and quantifiers are satisfied within one and the same valuation $\nu_{s}$.

In the domain of valuations which are supervaluations with respect to a certain partial valuation the axioms of classical logic remain valid. In particular, a revision of the tertium non datur is not required. Likewise, the usual definitions of logical truth and logical consequence are retained. It is only the notion of truth and falsehood which require a certain modification. In order to account for the difference between "real" values and assignments of arbitrary values, just filling the truth-value gaps, these notions are understood as follows:

E 10. Given is a partial valuation $\nu_{p}$ for the sentences of a language $L_{1} . S_{s}=$ $\left\{\nu_{s}(1), \nu_{s}(2), \ldots\right\}$ is the set of corresponding supervaluations.

i) $\phi$ is true iff $\nu_{s}(i)(\phi)=\mathrm{T}$ for every $\nu_{s}(i) \in S_{s}$.

ii) $\phi$ is false iff $\nu_{s}(i)(\phi)=\mathrm{F}$ for every $\nu_{s}(i) \in S_{s}$.

iii) $\phi$ is indeterminate iff there are natural numbers $k$ and $j$ such that $\nu_{s}(k)(\phi)$ $=\mathrm{T}$ and $\nu_{s}(j)(\phi)=\mathrm{F}$.

Note that there are not only sentences of $\Phi_{p}$ which have determinate values but also ones of $\operatorname{Sent}\left(L_{1}\right) \backslash \Phi_{p}$. More precisely, every sentence of $\operatorname{Sent}\left(L_{1}\right) \backslash \Phi_{p}$ that is a logical truth and every sentence of $\operatorname{Sent}\left(L_{1}\right) \backslash \Phi_{p}$ that is a logical consequence of $\left\{\phi \mid \nu_{p}(\phi)=\mathrm{T}\right\} \cup\left\{\neg \phi \mid \nu_{p}(\phi)=\mathrm{F}\right\}$ has a determinate value.

\section{Judging a Theory}

In this section, I will deal with the criteria according to which the epistemic quality of a system of postulates can be judged. The criteria obtained at the end of this section will, in the next section, be shown to be equivalent with the conditions under which the Ramsey sentence is true. As indicated in the introduction, the present paper aims at establishing another solution to the problem of theoretical terms, which is equivalent to the Ramsey solution. The claim that the two solutions are equivalent is understood in the sense that the criteria of judging a theory in the account being developed here are satisfied iff the Ramsey sentence is true.

It is common to say that a theory is true iff its axioms are true propositions in the intended domain of application. However, such an explanation is only applicable if the non-logical symbols occurring in the axioms are already interpreted. If, by contrast, the axioms are part of the interpretation of the non-logical symbols, as is the case with postulates and theoretical terms, then the truth-value assignment to the axioms of the theory implies no assertion about the factual adequacy of this theory. The rules of truth set up in E9 even exclude that a postulate is assigned to the value false. For this reason an alternative criteria to judge the epistemic quality of a theory is sought for. Though there is no 
direct criterion of truth in the sense of factual adequacy, an indirect assessment seems to be available, since the postulates ought to satisfy the requirement of consistency in the context of the whole system of truth-value assignments. Every deductive system is commonly subjected to this requirement for the simple reason that from an inconsistent set of sentences every arbitrary sentence is derivable. The notion of consistency is usually introduced by the following syntactic definition:

D 3. A set of sentences $\Phi$ is consistent as to derivations iff there is no sentence $\phi$ such that $\Phi \vdash \phi$ and $\Phi \vdash \neg \phi$.

In addition to the syntactic understanding, consistency with respect to semantic consequences is of particular interest. This notion is introduced by Alonzo Church (1956), p. 327, as follows:

D 4. A set of sentences $\Phi$ is consistent as to consequences iff there is no sentence $\phi$ such that $\Phi \models \phi$ and $\Phi \models \neg \phi$.

For reasons which will become obvious later on, the following considerations refer to the notion of consistency as to consequences. The relationship between both notions depends on whether the deductive system is sound and complete. In a deductive system that is complete and sound, the notions are equivalent. If the deductive system is sound but not complete, inconsistency as to consequences follows from inconsistency as to derivations but not vice versa.

I may proceed to apply the criterion of consistency to a formal system with postulates, assuming that E9 is adopted for the truth-value assignment to theoretical sentences. In such a system there are three types of sentences having the value true:

i) The sentences of $\Phi_{o}=\left\{\phi \mid \phi \in \operatorname{Sent}\left(L\left(V_{o}\right)\right)\right.$ and $\left.\mathfrak{A}_{o}=\phi\right\}$, where $\mathfrak{A}_{o}$ is the intended interpretation of the observational language. ${ }^{7}$

ii) The postulates $T C$.

iii) The sentences of $\Phi_{t}=\left\{\phi \mid \phi \in \operatorname{Sent}\left(L\left(V_{o}, V_{t}\right)\right) \backslash \operatorname{Sent}\left(L\left(V_{o}\right)\right) \backslash \Phi_{T C}\right.$ and $\Phi_{T C} \cup \Phi_{o}=\phi$ and $\Phi_{T C} \cup \Phi_{o} \cup\{\phi\}$ is satisfiable $\}$. $\Phi_{t}$ is the set of theoretical sentences that are not postulates and are assigned to the value true according to explanation E9.

If $\Phi_{o} \cup \Phi_{T C} \cup \Phi_{t}$ is consistent, then the theory can be accepted, if not, it has to be modified or discarded. It goes without saying that there may well be

\footnotetext{
${ }^{7}$ If there is more than one interpretation that satisfies an axiomatic system, then there might be one single interpretation such that the axioms of that system were set up to account for the truths of that interpretation. This interpretation is referred to by the term "intended interpretation". For example, there are several interpretations satisfying the Peano axioms, but only the natural numbers are considered as the intended domain of interpretation for the language in which that axiomatic system is formulated. Likewise, it is sensible to speak of an intended intended interpretation of an observational language. Such an interpretation is such that a certain truth-value assignment to the sentences of the formalized language results from it; it can be given by expressions of a non-formalized meta-language and it.
} 
different theories each being compatible with the observational facts in one and the same observational domain. That the rules adopted here do not determine the postulates of a theory to be true or false by empirical facts nicely squares with this well known consideration concerning the underdetermination of theory by the observational data.

The criterion just developed for judging a theory - that is, the requirement that $\Phi_{o} \cup \Phi_{T C} \cup \Phi_{t}$ is consistent - refers to a set of sentences that contains redundant information as there is a subset of $\Phi_{o} \cup \Phi_{T C} \cup \Phi_{t}$ that logically implies every sentence of that set. The same applies to the truth-rules for theoretical sentences in E9. Certainly, redundancy is not desirable for computational and notational reasons. Furthermore, transforming the criterion under consideration will turn out to be helpful to show that this criterion is satisfied iff the Ramsey sentence is true. By the following theorems we will accomplish a simplification of both the criterion for judging the theory and the truth-rules for theoretical sentences:

Th 1. $\Phi$ is satisfiable iff there is no sentence $\phi$ such that $\Phi \models \phi$ and $\Phi \models \neg \phi$.

Proof. For the left-to-right-direction, suppose $\Phi$ is satisfiable. Suppose furthermore, as assumption of an indirect proof, there is a sentence $\phi_{1}$ such that i) $\Phi$ $\models \phi_{1}$ and ii) $\Phi \models \neg \phi_{1}$. Since $\Phi$ is satisfiable, there is to be a structure $\mathfrak{A}_{1}$ such that $\mathfrak{A}_{1}=\Phi$, that means, every sentence of $\Phi$ is valid in $\mathfrak{A}_{1}$. Furthermore $\phi_{1}$ must either be true or false in $\mathfrak{A}_{1}$. To put it formally, either $\mathfrak{A}_{1} \models \phi_{1}$ or $\mathfrak{A}_{1} \models$ $\neg \phi_{1}$ must hold. In the first case, $\mathfrak{A}_{1}$ is a structure satisfying $\Phi$ and $\phi_{1}$, and thus $\Phi \cup \phi_{1}$ is satisfiable. So, in this case, $\Phi=\neg \phi_{1}$ does not hold. In the second case, $\mathfrak{A}_{1}$ is a structure satisfying $\Phi$ and $\neg \phi_{1}$, and thus $\Phi \cup \neg \phi_{1}$ is satisfiable. So, $\Phi \models \phi_{1}$ does not hold. In both cases we receive a contradiction, either to i) or to ii) above.

For the right-to-left direction, suppose there is no sentence $\phi$ such that i) $\Phi=\phi$ and ii) $\Phi \models \neg \phi$. As assumption of the indirect proof take: $\Phi$ is not satisfiable. If $\Phi$ is not satisfiable, then, trivially, every sentence $\phi$ is a semantic consequence of $\Phi$. Hence, there is a sentence $\phi_{1}$ such that i) $\Phi \models \phi_{1}$ and ii) $\Phi \models \neg \phi_{1}$. This is a contradiction to the assumptions. ${ }^{8}$

Th 2. $\Phi_{1}$ and $\Phi_{2}$ are sets of sentences such that every sentence of $\Phi_{2}$ is a logical consequence of $\Phi_{1}$. Then $\Phi_{1} \cup \Phi_{2}$ is satisfiable iff $\Phi_{1}$ is satisfiable.

Proof. For the left-to-right direction, assume that $\Phi_{1} \cup \Phi_{2}$ is satisfiable. Then there is a structure satisfying every sentence of $\Phi_{1}$ and every one of $\Phi_{2}$. So, trivially, $\Phi_{1}$ is satisfiable. For the right-to-left-direction, suppose $\Phi_{1}$ is satisfiable. Let $\mathfrak{A}_{1}$ be a structure satisfying every sentence of $\Phi_{1}$. Since every sentence of $\Phi_{2}$ is a logical consequence of $\Phi_{1}$, every sentence of $\Phi_{2}$ is valid in $\mathfrak{A}_{1}$ which follows from the definition of logical consequence. Hence, there is a structure satisfying $\Phi_{1} \cup \Phi_{2}$.

Th 3. $\Phi_{1}, \Phi_{2}$, and $\Phi_{3}$ are sets of sentences. $\Phi_{2} \subseteq \Phi_{3}$. Every sentence of $\Phi_{3}$ is a logical consequence of $\Phi_{2}$. Then $\Phi_{1} \cup \Phi_{2}=\phi$ iff $\Phi_{1} \cup \Phi_{3} \models \phi$.

\footnotetext{
${ }^{8}$ The theorem just proved is also stated by Church (1956), p. 328. The proof is nevertheless left to the reader.
} 
Proof. For the left-to-right direction, assume that $\Phi_{1} \cup \Phi_{2} \models \phi$. Then $\operatorname{Mod}(\phi) \subseteq$ $\operatorname{Mod}\left(\Phi_{1}\right) \cap \operatorname{Mod}\left(\Phi_{2}\right)$, where $\operatorname{Mod}(\Phi)$ stands for the set of structures satisfying the set of sentences $\Phi$. First we show that $\operatorname{Mod}\left(\Phi_{2}\right)=\operatorname{Mod}\left(\Phi_{3}\right)$. Assume that $\mathfrak{A}_{1}$ is a structure satisfying $\Phi_{2}$. Since every sentence of $\Phi_{3}$ is a logical consequence of $\Phi_{2}, \mathfrak{A}_{1}$ is also satisfying $\Phi_{3}$. Assume that $\mathfrak{A}_{2}$ is a structure satisfying $\Phi_{3}$. Since every sentence of $\Phi_{2}$ is a member of $\Phi_{3}, \mathfrak{A}_{2}$ is also satisfying $\Phi_{2}$. Hence, $\operatorname{Mod}\left(\Phi_{2}\right)=\operatorname{Mod}\left(\Phi_{3}\right)$. Since $\operatorname{Mod}(\phi) \subseteq \operatorname{Mod}\left(\Phi_{1}\right) \cap \operatorname{Mod}\left(\Phi_{2}\right)$ and $\operatorname{Mod}\left(\Phi_{2}\right)=$ $\operatorname{Mod}\left(\Phi_{3}\right)$, it holds that $\operatorname{Mod}(\phi) \subseteq \operatorname{Mod}\left(\Phi_{1}\right) \cap \operatorname{Mod}\left(\Phi_{3}\right)$. Thus, $\Phi_{1} \cup \Phi_{3} \models \phi$. The right-to-left direction is analogous to the left-to-right direction.

In the domain of observational sentences it is sufficient to consider just the atomic sentences instead of the whole set $\Phi_{o}$, the set of true sentences of $L\left(V_{o}\right)$. Let $\Phi_{a o}$ be the set containing every true atomic sentence of $L\left(V_{o}\right)$ and the negation of every false atomic sentence of this language. The interpretation of the observational language be restricted to canonical structures. This means, every individual of the domain in which $L\left(V_{o}\right)$ is interpreted must be designated by some constant of $V_{o}$ in an interpretation of that language. ${ }^{9}$ Then, we can prove the following theorem:

Th 4. $\mathfrak{A}_{o} \models \phi$ iff $\Phi_{a o}=\phi$, where $\phi$ is a sentence of $L\left(V_{o}\right)$ and $\mathfrak{A}_{o}$ the intended interpretation of $L\left(V_{o}\right)$.

Proof. For the left-to-right direct, suppose $\mathfrak{A}_{o} \models \phi_{1}$, where $\phi_{1}$ is a sentence of $L\left(V_{o}\right) . \mathfrak{A}_{o} \models \Phi_{a o}$ holds due to the definition of the set $\Phi_{a o}$. In the so called truth-value semantics the role of structures in the sense of model-theoretic semantics is taken over by truth-value assignments to the set of atomic sentences. Since the quantifiers are interpreted according to the substitutional reading, an atomic truth-value assignment determines uniquely the value of every sentence of the language. Although truth-value semantics is not adopted here, theorems concerning the relation between truth-value semantics and model-theoretic semantics will help to attain the intended result. If an atomic truth-value assignment $\nu$ determines a sentence $\phi$ to be true, this sentence is said to be true on $\nu$. A set of sentences $\Phi$ is said to be true on an atomic truth-value assignment $\nu$ iff every sentence of $\Phi$ is true on $\nu$. In the following I adhere to the version of truth-value semantics in which a sentence $\phi$ is said to be a logical consequence of a set of sentences $\Phi$ iff, for every atomic truth-value assignment $\nu$ on which $\Phi$ is true, $\phi$ is also true on $\nu{ }^{10}$ Furthermore, a set of sentences $\Phi$ is satisfiable in the truth-value sense iff there is an atomic truth-value assignment on which

\footnotetext{
${ }^{9}$ The notion of a canonical structures has been adopted from Barwise (1977), p. 31. Carnap himself requires that, for the observational language, every value of an individual variable is designated by an expression of $L\left(V_{o}\right)$. Carnap (1956), p. 41n. The restriction to canonical structures for the interpretation of $L\left(V_{o}\right)$ is therefore justified. Irrespective of Carnap's particular concept of an observational language it is reasonable to require for such a language that our linguistic means be sufficient to refer to every individual of the observational domain. For reasons of simplicity it is assumed in the present paper that every individual of the observational domain is designated by an individual constant. If there were individuals of the observational domain which are designated by a closed function expressions but not by an individual constant, the argumentation would remain valid, though it would have to be extended.

${ }^{10}$ This version is to be preferred if one aspires to have a kind of truth-value semantics being equivalent to the model-theoretic one in which the domain of interpretations is restricted to
} 
every sentence of $\Phi$ is true. Now, consider the valuation $\nu_{o}(\phi)=\mathrm{T}$ iff $\phi \in$ $\Phi_{a o}$ and $\nu_{o}(\phi)=\mathrm{F}$ iff $\neg \phi \in \Phi_{a o}$. Obviously, $\nu_{o}(\phi)$ is an atomic truth-value assignment. It is also called the truth-value counterpart of $\mathfrak{A}_{o}$. Now, there is a theorem saying that, for every canonical structure $\mathfrak{A}$ and every sentence $\phi, \mathfrak{A}$ $\models \phi$ iff $\phi$ is true on the truth-value counterpart of $\mathfrak{A}$. (See Leblanc (1976), p. 94.) Therefore, since, by supposition, $\mathfrak{A}_{o} \models \phi_{1}, \phi_{1}$ is also true on $\nu_{o}$. Since, obviously, $\nu_{o}$ is the only atomic truth-value assignment on which $\Phi_{a o}$ is true, it holds that, for every atomic truth-value assignment $\nu$ on which every sentence of $\Phi_{a o}$ is true, $\phi_{1}$ is also true on $\nu$. Hence, $\phi_{1}$ is a logical consequence of $\Phi_{a o}$ in the truth-value sense just defined. Therefore, $\Phi_{a o} \cup\left\{\neg \phi_{1}\right\}$ is not satisfiable in the truth-value sense. Now, $\Phi$ is satisfiable in the truth-value sense iff there is a canonical structure satisfying $\Phi$. (See Leblanc (1976), p. 94.) Therefore, since $\Phi_{a o} \cup\left\{\neg \phi_{1}\right\}$ is not satisfiable in the truth-value sense, $\Phi_{a o} \models \phi_{1}$ holds also in the model-theoretic sense, given the interpretation of the observational language is restricted to canonical structures.

For the right-to-left direction, suppose $\Phi_{a o} \models \phi_{1}$. Let $\nu_{o}$ be, like above, the truth-value counterpart of $\mathfrak{A}_{o}$. Since $\Phi_{a o} \models \phi_{1}$ holds in the model-theoretic sense, $\phi_{1}$ is also a logical consequence of $\Phi_{a o}$ in the truth-value sense. Then, by $\Phi_{a o}$ being true on $\nu_{o}, \phi_{1}$ is true on $\nu_{o}$. There is a theorem saying that, for every canonical structure $\mathfrak{A}$ and the corresponding truth-value counterpart $\nu, \mathfrak{A}=\phi$ iff $\phi$ is true on $\nu$. (See Leblanc (1976), p. 94.) Hence, $\mathfrak{A}_{o} \models \phi_{1}$.

How do the preceding theorems contribute to a simplification of the truth-rules for theoretical sentences? By Th4, every sentence of $\Phi_{o}$, the set of true $L\left(V_{o}\right)$ sentences, is a logical consequence of $\Phi_{a o}$. Th3 therefore applies to the sets $\Phi_{T C}$ $\cup \Phi_{o}$ and $\Phi_{T C} \cup \Phi_{a o}$. Hence, $\Phi_{T C} \cup \Phi_{o} \models \phi$ iff $\Phi_{T C} \cup \Phi_{a o} \models \phi$. By analogous reasons, Th2 applies to the sets $\Phi_{T C} \cup \Phi_{o} \cup\{\phi\}$ and $\Phi_{T C} \cup \Phi_{a o} \cup\{\phi\}$. Hence, $\Phi_{T C} \cup \Phi_{o} \cup\{\phi\}$ is satisfiable iff $\Phi_{T C} \cup \Phi_{a o} \cup\{\phi\}$ is satisfiable. This being the case, the truth-rules in E9 admit of the following simplification:

E 9.1. $\nu_{t}(\phi)$ is a partial valuation for sentences of $\operatorname{Sent}\left(L\left(V_{o}, V_{t}\right)\right) \backslash \operatorname{Sent}\left(L\left(V_{o}\right)\right)$ $\backslash \Phi_{T C}$.

i) $\nu_{t}(\phi)=\mathrm{T}$ iff $\Phi_{T C} \cup \Phi_{a o}=\phi$ and $\Phi_{T C} \cup \Phi_{a o} \cup\{\phi\}$ is satisfiable.

ii) $\nu_{t}(\phi)=\mathrm{F}$ iff $\Phi_{T C} \cup \Phi_{a o} \models \neg \phi$ and $\Phi_{T C} \cup \Phi_{a o} \cup\{\neg \phi\}$ is satisfiable.

Hence, $\Phi_{t}$, the set of true sentences at the theoretical level, is equivalent to the set $\left\{\phi \mid \phi \in \operatorname{Sent}\left(L\left(V_{o}, V_{t}\right)\right) \backslash \operatorname{Sent}\left(L\left(V_{o}\right)\right) \backslash \Phi_{T C}\right.$ and $\Phi_{T C} \cup \Phi_{a o} \models \phi$ and $\Phi_{T C}$ $\cup \Phi_{a o} \cup\{\phi\}$ is satisfiable $\}$.

How do the preceding theorems contribute to simplify our criterion for judging a theory in the sense of the dual-level conception? By Th1, $\Phi_{T C} \cup \Phi_{o} \cup \Phi_{t}$ is

canonical structures. It is adopted by Stegmüller in his (1984), p. 84, but rejected by Leblanc in his (1976), p. 17n. Leblanc, unlike Stegmüller, intends to have a truth-value semantics that is equivalent to the model-theoretic one without restriction to canonical structures. (Leblanc uses the term "Henkin structure" to refer to interpretations that are, in the present paper, called canonical structures. See Leblanc (1976), p. 20.) The reason for my adherence to Stegmüller's version is that the interpretation of $L\left(V_{o}\right)$ is restricted to canonical structures. This restriction has been explicitly mentioned as a premise of the theorem currently to be proved. 
consistent as to consequences iff $\Phi_{T C} \cup \Phi_{o} \cup \Phi_{t}$ is satisfiable. By Th4, every sentence of $\Phi_{o}$ is a logical consequence of $\Phi_{a o}$. Th2 therefore applies to the sets $\Phi_{T C} \cup \Phi_{o} \cup \Phi_{t}$ and $\Phi_{T C} \cup \Phi_{a o} \cup \Phi_{t}$. Hence, $\Phi_{T C} \cup \Phi_{o} \cup \Phi_{t}$ is satisfiable iff $\Phi_{T C} \cup \Phi_{a o} \cup \Phi_{t}$ is satisfiable. Since, by E9.2, every sentence of $\Phi_{t}$ is a logical consequence of $\Phi_{T C} \cup \Phi_{a o}$, Th2 applies to the sets $\Phi_{T C} \cup \Phi_{a o} \cup \Phi_{t}$ and $\Phi_{T C} \cup$ $\Phi_{a o}$. Hence, $\Phi_{T C} \cup \Phi_{a o} \cup \Phi_{t}$ is satisfiable iff $\Phi_{T C} \cup \Phi_{a o}$ is satisfiable. To sum, $\Phi_{T C} \cup \Phi_{o} \cup \Phi_{t}$ is consistent as to consequences iff $\Phi_{T C} \cup \Phi_{a o}$ is satisfiable. So, the considerations of the present section may be summarized by the following proposition:

$\mathbf{P}$ 2. $T C$ is a theory in the sense of the dual-level conception of scientific language. $\mathfrak{A}_{o}$ is the intended interpretation of the observational language $L\left(V_{o}\right)$. $\Phi_{a o}$ is the set that contains every atomic sentence of $L\left(V_{o}\right)$ being true in $\mathfrak{A}_{o}$ and the negation of every atomic sentence of $L\left(V_{o}\right)$ being false in $\mathfrak{A}_{o}$. E9 is adopted as rules for the truth-value assignment to theoretical sentences. Then the postulates $T C$ cannot be assigned to the value false whatever the empirical facts may be. The theory $T C$ is rather subject to the following two equivalent criteria:

i) $\Phi_{T C} \cup \Phi_{a o}$ is satisfiable.

ii) $\Phi_{T C} \cup \Phi_{a o}$ is consistent as to consequences.

The equivalence of these criteria follows from Th1.

\section{Comparison to the Ramsey Account}

By a careful examination of the notion of an indirect interpretation we have seen that the postulates of a theory are to be understood as imposing a constraint on the admissible valuations of theoretical terms and sentences. Since, on this reading of postulates, the problem of theoretical terms does not arise, the present account amounts to a second solution to the problem of theoretical terms. This solution is equivalent to the Ramsey solution in the sense that the criteria for judging a system of postulates, as given by proposition P2, are satisfied iff the corresponding Ramsey sentence is true:

Th 5. The Ramsey sentence $T C^{R}$ is true iff $\Phi_{T C} \cup \Phi_{a o}$ is satisfiable, where $\Phi_{a o}$ is a sentential representation of the observational facts, as explained in P2.

Proof. For the left-to-right direction, suppose $T C^{R}$ is true. This means, $\left(D_{o}\right.$, $\left.D_{t}, \mathfrak{a}_{o}\right) \models \exists X_{1} \ldots \exists X_{n} T C\left(X_{1}, \ldots, X_{n}, n_{1}, \ldots, n_{k}\right)$, where $D_{o}$ is the domain of interpretation of the observable language, $D_{t}$ the domain of interpretation of the theoretical language, and $\mathfrak{a}_{o}$ a function assigning extensional interpretations to the predicate and function symbols and the individual constants. $\left(D_{o}, \mathfrak{a}_{o}\right)=$ $\mathfrak{A}_{o}$ is the intended interpretation of the observational language. $\mathfrak{A}_{o} \models \Phi_{\text {ao }}$ holds by definition of $\Phi_{a o}$. The truth of $T C^{R}$ amounts to the fact that there is an extensional interpretation of the non-logical symbols of the theoretical vocabulary on which the postulates $T C$ are true, where the observational vocabulary is interpreted according to $\mathfrak{a}_{o}$. Call this interpretation $\left(\mathfrak{a}_{o / t}, \mathfrak{a}_{t}\right)$, where $\mathfrak{a}_{o / t}$ stands 
for the extensional interpretation of theoretical terms with "mixed" argument structure, i. e. argument structures having places for individuals of $D_{o}$ and $D_{t}$, whereas $\mathfrak{a}_{t}$ is the interpretation of theoretical terms whose argument structure admits no individuals of $D_{o}{ }^{11}$ Hence, $\left(D_{o}, D_{t}, \mathfrak{a}_{o}, \mathfrak{a}_{o / t}, \mathfrak{a}_{t}\right) \models T C$. Since the truth-value assignment to sentences of $L\left(V_{o}\right)$ is not affected by the interpretation of the theoretical vocabulary, $\left(D_{o}, D_{t}, \mathfrak{a}_{o}, \mathfrak{a}_{o / t}, \mathfrak{a}_{t}\right) \models \Phi_{a o}$ holds in the light of $\mathfrak{A}_{o} \models \Phi_{a o}$. Hence, $\left(D_{o}, D_{t}, \mathfrak{a}_{o}, \mathfrak{a}_{o / t}, \mathfrak{a}_{t}\right) \models \Phi_{a o} \cup \Phi_{T C}$. Therefore $\Phi_{a o} \cup \Phi_{T C}$ is satisfiable.

For the left-to-right-direction, suppose $\Phi_{a o} \cup \Phi_{T C}$ is satisfiable. Hence, there is a structure $\left(D_{o 1}, D_{t 1}, \mathfrak{a}_{o 1}, \mathfrak{a}_{o 1 / t 1}, \mathfrak{a}_{t 1}\right)=\Phi_{a o} \cup \Phi_{T C}$, call it $\mathfrak{A}_{1}$. Since $\left(D_{o 1}, \mathfrak{a}_{o 1}\right)$ may well be distinct from $\left(D_{o}, \mathfrak{a}_{o}\right)$, this does not immediately imply the truth of $T C^{R}$. It is therefore necessary to construct from $\mathfrak{A}_{1}$ a structure for $L\left(V_{o}, V_{t}\right)$ which agrees with $\mathfrak{a}_{o}$ on the interpretation of the observational vocabulary. Now, let $\pi$ be a function mapping individuals of $D_{o 1}$ to those of $D_{o}$ according the following equation:

(7) $\mathfrak{a}_{o}(c)=\pi\left(\mathfrak{a}_{o 1}(c)\right)$ for every individual constant $c$ of $L\left(V_{o}\right)$.

So, $\pi$ is defined by the requirement that, if the constant $\mathrm{c}$ designates an individual a on $\mathfrak{a}_{o 1}$, then the value of $\pi(\mathrm{a})$ is the individual designated by $\mathrm{c}$ on $\mathfrak{a}_{o}$. Since $\left(D_{o}, \mathfrak{a}_{o}\right)$ is a canonical structure, every individual of $D_{o}$ belongs to the domain of values of $\pi$. Hence, $\pi$ is surjective. Furthermore, $\pi$ is injective iff from $\pi\left(\mathfrak{a}_{o 1}\left(c_{i}\right)\right)=\pi\left(\mathfrak{a}_{o 1}\left(c_{j}\right)\right)$ it follows that $\mathfrak{a}_{o 1}\left(c_{i}\right)=\mathfrak{a}_{o 1}\left(c_{j}\right)$ for every pair of individual constants $\left(c_{i}, c_{j}\right)$ of $L\left(V_{o}\right)$. Suppose $\pi\left(\mathfrak{a}_{o 1}\left(c_{i}\right)\right)=\pi\left(\mathfrak{a}_{o 1}\left(c_{j}\right)\right)$ for an arbitrary, but determinate pair $\left(c_{i}, c_{j}\right)$. Hence, by $\mathfrak{a}_{o}(\mathrm{c})=\pi\left(\mathfrak{a}_{o 1}(\mathrm{c})\right), \mathfrak{a}_{o}\left(c_{i}\right)$ $=\mathfrak{a}_{o}\left(c_{j}\right)$. Now, $\mathfrak{a}_{o}\left(c_{i}\right)=\mathfrak{a}_{o}\left(c_{j}\right)$ iff the atomic sentence $c_{i}=c_{j}$ is true on $\mathfrak{a}_{o}$. Analogously, $\mathfrak{a}_{o 1}\left(c_{i}\right)=\mathfrak{a}_{o 1}\left(c_{j}\right)$ iff the sentence $c_{i}=c_{j}$ is true on $\mathfrak{a}_{o 1}$. Since $\mathfrak{a}_{o}$ and $\mathfrak{a}_{o 1}$ agree on the values of the atomic sentences and $L\left(V_{o}\right)$ contains a sign for identity, $\mathfrak{a}_{o}\left(c_{i}\right)=\mathfrak{a}_{o}\left(c_{j}\right)$ iff $\mathfrak{a}_{o 1}\left(c_{i}\right)=\mathfrak{a}_{o 1}\left(c_{j}\right)$. Then, from $\mathfrak{a}_{o}\left(c_{i}\right)=\mathfrak{a}_{o}\left(c_{j}\right)$ it follows that $\mathfrak{a}_{o 1}\left(c_{i}\right)=\mathfrak{a}_{o 1}\left(c_{j}\right)$. Hence, $\pi$ is injective. So, since $\pi$ is also surjective, $\pi$ is bijective. Now, consider the function $\pi_{c}: D_{o 1} \cup D_{t 1} \rightarrow D_{o} \cup D_{t}$ :

(8) $\quad \pi_{c}=\left\{\pi_{c}(\mathrm{a})=\pi(\mathrm{a})\right.$ for every individual a of $D_{o 1}$ and $\pi_{c}(\mathrm{a})=$ a for every individual a of $\left.D_{t 1}\right\}$.

Note that $D_{t 1}=D_{t}$, since there is, in Carnap's version of the dual-level conception, no variation in the domain of interpretation of the $V_{t}$ symbols, the non-logical symbols of the theoretical vocabulary. Obviously, $\pi_{c}$ is also bijective. Through the help of $\pi_{c}$ we construct a structure $\mathfrak{A}_{2}=\left(D_{o}, D_{t}, \mathfrak{a}_{o 2}, \mathfrak{a}_{o 2 / t 2}\right.$, $\left.\mathfrak{a}_{t 2}\right)$ :

i) $\mathfrak{a}_{o 2}(\mathrm{c})=\pi_{c}\left(\mathfrak{a}_{o 1}(\mathrm{c})\right)$ for every individual constant c of $L\left(V_{o}\right)$.

\footnotetext{
${ }^{11}$ It may well happen that there is a function that takes empirical objects as arguments and has individuals of the theoretical domain as values. To give an example, we need such functions to express formally that a particular empirical object has a certain mass. The necessity to distinguish between theoretical terms with mixed and pure theoretical argument structure is pointed out by Ketland (2004), p. 290.
} 
ii) $\left(\pi_{c}\left(a_{1}\right), \ldots, \pi_{c}\left(a_{n}\right)\right) \in \mathfrak{a}_{o 2}(\mathrm{P})$ iff $\left(a_{1}, \ldots, a_{n}\right) \in \mathfrak{a}_{o 1}(\mathrm{P})$ for every n-ary predicate symbol $\mathrm{P}$ of $L\left(V_{o}\right)$ and $a_{1}, \ldots, a_{n} \in D_{o 1}$.

iii) $\mathfrak{a}_{o 2}(f)\left(\pi_{c}\left(a_{1}\right), \ldots, \pi_{c}\left(a_{n}\right)\right)=\pi_{c}\left(\mathfrak{a}_{o 1}(f)\left(a_{1}, \ldots, a_{n}\right)\right)$ for every n-ary function symbol $f$ of $L\left(V_{o}\right)$ and $a_{1}, \ldots, a_{n} \in D_{o 1}$.

iv) $\left(\pi_{c}\left(a_{1}\right), \ldots, \pi_{c}\left(a_{k}\right), \pi_{c}\left(a_{1}\right), \ldots, \pi_{c}\left(a_{n}\right)\right) \in \mathfrak{a}_{o 2 / t 2}(\mathrm{P})$ iff $\left(a_{1}, \ldots, a_{k}, a_{1}, \ldots, a_{n}\right)$ $\in \mathfrak{a}_{o 1 / t 1}(\mathrm{P})$ for every $(\mathrm{n}+\mathrm{k})$-ary predicate symbol $\mathrm{P}$ of $L\left(V_{o}, V_{t}\right)$ with mixed argument structure, where $a_{1}, \ldots, a_{k} \in D_{o 1}$ and $a_{1}, \ldots, a_{n} \in D_{t}$.

v) $\mathfrak{a}_{o 2}(f)\left(\pi_{c}\left(a_{1}\right), \ldots, \pi_{c}\left(a_{k}\right), \pi_{c}\left(a_{1}\right), \ldots, \pi_{c}\left(a_{n}\right)\right)=\pi_{c}\left(\mathfrak{a}_{o 1}(f)\left(a_{1}, \ldots, a_{k}, a_{1}, \ldots, a_{n}\right)\right)$ for every $(\mathrm{k}+\mathrm{n})$-ary function symbol $f$ of $L\left(V_{o}, V_{t}\right)$ with mixed argument structure, where $a_{1}, \ldots, a_{k} \in D_{o 1}$ and $a_{1}, \ldots, a_{n} \in D_{t}$.

vi) $\mathfrak{a}_{t 2}=\mathfrak{a}_{t 1}$.

$\mathfrak{a}(\alpha)$ designates the interpretation of the symbol $\alpha$ according to $\mathfrak{a}$, where $\alpha$ can be a constant, a predicate or a function symbol. Since $\pi_{c}$ is an isomorphism from $D_{o 1} \cup D_{t 1}$ to $D_{o} \cup D_{t}$, the construction rules i) to vi) guarantee that $\mathfrak{A}_{1}$ and $\mathfrak{A}_{2}$ are isomorphic. Since in isomorphic structures the same sentences are true, it follows from $\mathfrak{A}_{1}=\Phi_{a o} \cup \Phi_{T C}$ that $\mathfrak{A}_{2} \models \Phi_{a o} \cup \Phi_{T C}$. Hence, there is an extensional interpretation of the theoretical terms on which the postulates are true sentences, where the observational terms are interpreted according to $\mathfrak{a}_{o}$. Hence, $T C^{R}$.

The Ramsey sentence thus turns out to be equivalent to a proposition of the meta-language stating that the postulates are semantically compatible with a sentential representation of the observational facts. ${ }^{12}$ Hence, there are, arguably, two accounts of the semantic peculiarities of theoretical terms. The one in the present paper is deeply inspired by Carnap's Logical Syntax of Language. The truth-rules for theoretical sentences are developed under the assumption that the postulates are set up as P-Grundsätze in the sense of this work. PGrundsätze, or P-rules in the English edition, are admitted to have empirical content, yet their truth-value is neither determined by the interpretation of the non-logical symbols nor by the values of atomic sentences. The present account may therefore be called the Logical Syntax of Language account of a scientific theory, or shorter, the LSL account.

\section{The Logical Status of Postulates}

There is, according to Stegmüller, a close connection between the problem of theoretical terms and there being sentences in most scientific theories which are neither factual statements nor definitions. Such sentences, so Stegmüller

\footnotetext{
${ }^{12}$ That the Ramsey sentence is equivalent to a proposition of the meta-language is not a completely new insight. Ketland has proved that $T C^{R}$ to a the claim that $\mathfrak{A}_{o}$, the intended interpretation of $L\left(V_{o}\right)$, can be expanded to an $L\left(V_{o}, V_{t}\right)$ structure satisfying the postulates. See Ketland (2004), p. 293. However, Ketland assumes a direct interpretation of the theoretical terms and thus makes no contribution to the problem under consideration in the present paper.
} 
(1976), p. 11 argues, cannot be interpreted as true statements in the sense of predicate logic. With respect to the truth-rules of standard semantics, this argumentation seems to be fully justified. In standard model-theoretic semantics the truth-value of a logical complex sentence that does not qualify as a definition is determined by the interpretation of the non-logical symbols. This kind of truth-value assignment implies, as shown above, the semantic circle that Sneed has termed the problem of theoretical terms.

I have hereby identified standard semantics with model-theoretic semantics and furthermore I assumed that, in any application of model-theoretic semantics to an empirical domain, the relevant fragment of language has one and only one intended interpretation representing the state of the domain. The uniqueness of interpretation, at least from the perspective of a single speaker, is a common convention in linguistics and philosophy of language. Now, if there is one and only one structure assumed to represent the state of the relevant domain, then the following division of sentences is complete:

i) Synthetic sentences, i. e. sentences which are true in, at least, one structure and false in, at least, another.

ii) Logical truths and logical falsehoods, i. e. sentences which are either true in every structure or false in every structure.

iii) Sentences having the status of a definition.

Such a classification leaves no room indeed for a semantically satisfying interpretation of postulates in predicate logic. The account developed here is, consequently, based on a rejection of the uniqueness assumption, that is, the assumption that the state of the world, or a fragment of it, can be represented by one and only one structure. This assumption remains valid only with respect to the observational language. By contrast, in the whole language $L\left(V_{o}, V_{t}\right)$, every structure that is an extension of the intended interpretation of the observational language and satisfies the postulates is considered to represent the state of the world, or a fragment of it.

It is illuminating if postulates are seen as semantically consistent extensions of the language. More precisely, postulates are required to extend the language in a consistent manner. Since the meaning of theoretical terms is introduced by postulates, the postulates extend the language. Nevertheless, since postulates may have and do have empirical consequences, they are, unlike definitions, not required to be conservative extensions of the language. Consistency is rather appropriate to require from postulates. This characterization nicely squares with a remark of Carnap (1975), p. 82, concerning the twofold function of postulates:

They [the postulates, H. A.] not only contribute to the meanings of the T-terms but they also set forth the factual content of the theory. This is evident from the fact that with their help, along with sentences on observable processes, predictions can be derived of futu re observable processes. Hence, a C-postulate or a T-postulate 
cannot in general (aside from certain special cases which we here leave aside) be taken as an A-postulate [i.e. an analytic sentence not being logically true, H.A.].

\section{References}

Andreas, H. (2007). Carnaps Wissenschaftslogik. Eine Untersuchung zur Zweistufenkonzeption. mentis, Paderborn.

Barwise, J. (ed.) (1977). Handbook of Mathematical Logic. North-Holland Publishing Company, Amsterdam.

Carnap, R. (1934). Logische Syntax der Sprache. Springer-Verlag, Wien.

Carnap, R. (1937). The Logical Syntax of Language. Routledge, London.

Carnap, R. (1939). Foundations of Logic and Mathematics. University of Chicago Press, Chicago.

Carnap, R. (1942). Introduction to Semantics. Harvard University Press, Cambridge, Mass.

Carnap, R. (1956). The Methodological Character of Theoretical Concept. In: H. Feigel and M. Scriven (eds.) Minnesota Studies in the Philosophy of Science I, University of Minnesota Press, Minneapolis, 38-76.

Carnap, R. (1958). Beobachtungssprache und theoretische Sprache. Dialectica 12: $236-248$.

Carnap, R. (1975). Observational Language and Theoretical Language. In: J. Hintikka (ed.) Rudolf Carnap. Logical Empiricist, D. Reidel Publishing Company, Dordrecht, 75-85.

Church, A. (1956). Introduction to Mathematical Logic. Princeton University Press, Princeton.

Dummett, M. (1978). Realism. In: Truth and Other Enigmas, Harvard University Press, Cambridge, Mass., chap. 10, 145-165.

Hempel, C. G. (1965). The Theoretician's Dilemma. In: Aspects of Scientific Explanation, Collier Macmillan Publishers, New York-London.

Ketland, J. (2004). Empirical Adequacy and Ramsification. British Journal for Philosophy of Science 55: 287-300.

Leblanc, H. (1976). Truth-Value Semantics. North Holland Publishing Company, Amsterdam.

Priest, G. (2001). An Introduction to Non-Classical Logic. Cambridge University Press, Cambridge. 
Ramsey, F. P. (1950). Theories. In: R. B. Braithwaite (ed.) The Foundations of Mathematics and Other Logical Essays, Humanities Press, New York, 212236.

Sneed, J. (1979). The Logical Structure of Mathematical Physics. D. Reidel Publishing Company, Dordrecht.

Stegmüller, W. (1970). Probleme und Resultate der Wissenschaftstheorie und Analytischen Philosophie. Bd. 2 - Theorie und Erfahrung, Teilbd. 1. SpringerVerlag, Berlin-Heidelberg-New York.

Stegmüller, W. (1976). The Structure and Dynamics of Theories. SpringerVerlag, Berlin-Heidelberg-New York.

Stegmüller, W. (1984). Strukturtypen der Logik. Springer-Verlag, BerlinHeidelberg-New York.

Stegmüller, W. (1985). Probleme und Resultate der Wissenschaftstheorie und Analytischen Philosophie. Bd. 2 - Theorie und Erfahrung, Teil D. SpringerVerlag, Berlin-Heidelberg-New York.

Tuomela, R. (1973). Theoretical Concepts. Springer-Verlag, Wien.

van Fraassen, B. (1969). Presuppositions, Supervaluations and Free Logic. In: K. Lambert (ed.) The Logical Way of Doing Things, Yale University Press, New Haven, 67-92. 